\title{
ON THE ANALYSIS AND SYNTHESIS OF CERTAIN ABSTRACT SYSTEMS
}

\author{
BY REESE T. PROSSER
}

Communicated by M. H. Protter, December 14, 1970

\begin{abstract}
The systems considered here are those describable as continuous functions mapping one compact metric space into another. A class of such systems is determinable if the members can be distinguished to within a given error by a finite number of elementary measurements made on the domain and range spaces. Such classes are necessarily relatively compact in the uniform metric and admit a common modulus of continuity. This announcement provides quantitative estimates of the number of measurements required in terms of the common modulus of continuity of the class, and the metric entropy, capacity, and dimension of the domain and range spaces. These data are used to construct uniform simplicial and polynomial approximations for each member of the class, and to provide quantitative estimates for the degrees of the approximating polynomials required.
\end{abstract}

For many purposes a physical system can be usefully modeled as a transfer function of prescribed continuity taking one metric space (the input space) into another (the output space). Within this framework, an analysis of the system is an approximate description of this function in terms of data taken from the input and output spaces, while a synthesis of the system is an approximate realization of the function in terms of known elementary functions. One primary purpose of systems studies is to provide effective analysis and synthesis procedures for rendering heretofore intractable systems accessible to modern computing machinery. We announce here some recent quantitative results bearing on the complexity of such procedures; details will appear elsewhere [1].

We denote by $X$ and $Y$ the input and output spaces, respectively. We suppose that both $X$ and $Y$ are furnished with a metric, which provides a measure of the distinguishability of elements in each of these spaces. We assume that the elements of both $X$ and $Y$ can be approximated to within any prescribed error by a finite number of elements; this assumption forces both $X$ and $Y$ to be (relatively) compact metric spaces [2].

AMS 1969 subject classifications. Primary 5425, 5470, 4141, 4160, 4115, 9310; Secondary $9410,9420$.

Key words and phrases. Systems analysis, compact metric spaces, approximate dimension, Kolmogorov widths, metric entropy, polynomial approximation. 
By a measurement on $X$ we mean any continuous real-valued function defined on $X$.

How many measurements are required to distinguish the elements of $X$ to within a prescribed error? We know that if $X$ has topological dimension $n$ then $2 n+1$ measurements will always suffice [3]. More generally, if $\varphi: X \rightarrow X^{\prime}$ is any continuous function from $X$ to another metric space $X^{\prime}$, then we define the resolution $\rho(\varphi)$ of $\varphi$ by

$$
\rho(\varphi)=\sup \left\{d\left(x, x^{\prime}\right): \varphi(x)=\varphi\left(x^{\prime}\right)\right\} .
$$

Evidently if $X^{\prime}$ has topological dimension $n$ and $\varphi$ has resolution $\rho(\varphi) \leqq \epsilon$, then $2 n+1$ measurements will again suffice. Hence we define, for each $\epsilon>0$, the $\epsilon$-dimension $d(\epsilon, X)$ of $X$ by

$$
d(\epsilon, X)=\inf \{\operatorname{dim} \varphi(X): \rho(\varphi) \leqq \epsilon\}
$$

where the infimum is taken over all continuous mappings $\varphi$ of $X$ into the Hilbert cube. Since $X$ is compact, $d(\epsilon, X)$ is finite for every $\epsilon>0$, and evidently $d(\epsilon, X) \uparrow$ as $\epsilon \downarrow 0$. In computing $d(\epsilon, X)$ it is useful to know that if $X$ contains an isometric copy of an $n$-dimensional $\epsilon$-ball, then $d(2 \epsilon, X) \geqq n$, [4], while if $X$ is contained isometrically in some Banach space, then

$$
d(2 \epsilon, X) \leqq \sup \left\{n: w_{n-1} \geqq \epsilon\right\}
$$

where $w_{n}$ are the Kolmogorov widths of $X$ [5]. If we write $b(\epsilon, X)$ for the largest $b$ such that $X$ contains a $b$-dimensional $\epsilon$-ball, and $n(\epsilon, X)$ as the largest $n$ for which $w_{n-1} \geqq \epsilon$, then

$$
b(\epsilon, X) \leqq d(2 \epsilon, X) \leqq n(\epsilon, X) .
$$

How many elements in $X$ are required to approximate every element in $X$ to within a prescribed error? Following Kolmogorov [6], we define, for each $\epsilon>0$, an $\epsilon$-net in $X$ as a subset $\left\{x_{i}\right\}$ such that, for each $x \in X, \min \left\{d\left(x, x_{i}\right)\right\}<\epsilon$. Since $X$ is compact, there are always finite $\epsilon$-nets in $X$. An $\epsilon$-net is optimal if it contains the least possible number of elements; we denote this number by $N(\epsilon, X)$ and its logarithm $\log _{2} N(\epsilon, X)$ by $H(\epsilon, X)$, the (relative) $\epsilon$-entropy of $X$. Similarly we define, for each $\epsilon>0$, an $\epsilon$-distinguished set for $X$ as a subset $\left\{x_{i}\right\}$ such that $\min \left\{d\left(x_{i}, x_{j}\right): i \neq j\right\}>\epsilon$. Since $X$ is compact, every $\epsilon$-distinguished set in $X$ is finite. An $\epsilon$-distinguished set is optimal if it contains the greatest possible number of elements; we denote this number by $M(\epsilon, X)$, and its logarithm $\log _{2} M(\epsilon, X)$ by $C(\epsilon, X)$, the $\epsilon$-capacity of $X$. The basic relations between these concepts are summarized in [6];

$$
H(2 \epsilon, X) \leqq C(2 \epsilon, X) \leqq H(\epsilon, X) .
$$


If we wish to approximate every element of $X$ to within $\epsilon$, we shall evidently need to construct at least $2^{c(2 e, X)}$ elements, and at most $2^{H(\epsilon, X)}$ in $X$.

The relations between these concepts and that of the $\epsilon$-dimension are less accessible. We know that $X$ always admits an equivalent metric for which

$$
b(4 \epsilon, X) \leqq H(2 \epsilon, X) \leqq C(2 \epsilon, X) \leqq d(\epsilon, X) \log 4(1+1 / \epsilon)
$$

and that this equivalent metric is assumed if $X$ is a compact subset of some Banach space [7].

By a system we mean any continuous function $f: X \rightarrow Y$. We associate with each such system a modulus of continuity $\omega$, defined by

$$
\omega(\delta, f)=\sup \left\{d\left(f(x), f\left(x^{\prime}\right)\right): d\left(x, x^{\prime}\right)<\delta\right\}
$$

which measures the continuity of $f$. For a given modulus of continuity $\omega$, we denote by $D$ the set of all systems $g$ whose moduli of continuity are dominated by $\omega$ :

$$
D=\{g: \omega(\delta, g) \leqq \omega(\delta)\} .
$$

We provide $D$ with the uniform metric:

$$
d(g, h)=\sup \{d(g(x), h(x)): x \in X\}
$$

and note that, by Arzela's theorem, $D$ is compact in this metric. As a consequence, the concepts of $\epsilon$-dimension, $\epsilon$-entropy and $\epsilon$-capacity can be introduced for $D$, and their magnitudes can be estimated in terms of the magnitudes of the corresponding concepts for $X$ and $Y$. For this purpose we define, for each $\epsilon>0$,

$$
\delta=\delta(\epsilon)=\sup \left\{\delta^{\prime}: \omega\left(\delta^{\prime}\right) \leqq \epsilon\right\}
$$

and prove:

Theorem 1 [1]. For $X, Y$ and $D$ as above, we have

$$
\begin{gathered}
H(2 \epsilon, D) \leqq H(\epsilon, Y) 2^{H(\delta, X)}, \\
b(\epsilon, Y) 2^{C(\delta, X)} \leqq C(2 \epsilon, D), \\
d(3 \epsilon, D) \leqq d(\epsilon, Y) 2^{H(\delta, X)}, \\
b(\epsilon, Y) 2^{C(\delta, X)} \leqq d(2 \epsilon, D) .
\end{gathered}
$$

These results provide us with useful estimates for the magnitude of complexity of a system. Given any system $f$, we first form the class $D$ according to $(7)$, with $\omega(\delta)=\omega(\delta, f)$. Then $H(\epsilon, D)$ and $C(\epsilon, D)$ provide upper and lower bounds for the number of different candidates in $D$ which we must examine in order to find one uniformly 
within $\epsilon$ of $f$; and $n(\epsilon, D)$ provides a lower bound for the dimension of any space of elementary systems which we must use in order to reconstruct $f$ uniformly to within $\epsilon$.

In order to approximate $f$ by elementary systems, we must first approximate $X$ and $Y$ by elementary spaces:

THEOREM 2. For each $\delta>0$, there exists a contraction mapping $\varphi: X \rightarrow X_{m}$, of resolution $\rho(\varphi) \leqq 5 \delta$, into a simplicial complex $X_{m}$ of dimension $m=d(\delta, X)$ with at most $(m+1) N(\delta, X)$ vertices lying in general position in $R^{2 m+1}$.

If $\delta, m, X_{m}$ and $\varphi: X \rightarrow X_{m}$ are constructed from $X$ as in Theorem 2, and $\epsilon=\omega(\delta), n=n(\epsilon, Y), Y_{n}$ and $\psi: Y \rightarrow Y_{n}$ are similarly constructed from $Y$, then we put

$$
D_{m n}=\left\{g: X_{m} \rightarrow Y_{n}: \omega(\delta, g) \leqq \omega(\delta)\right\}
$$

and search for approximate realizations for $f$ in $D_{m n}$.

Theorem 3. For each $\epsilon>0$ and for each $f \in D$, there exists $g \in D_{m n}$ such that $\omega(\delta, g) \leqq \omega(\delta, f)$, and

$$
d(\psi \circ f, g \circ \varphi) \leqq 9 \epsilon .
$$

It follows from this result that any $\epsilon$-net for $D_{m n}$ provides a $10 \epsilon$-net for $D$, and hence that

$$
H(10 \epsilon, D) \leqq H\left(\epsilon, D_{m n}\right) .
$$

On the other hand, $D_{m n}$ contains an $\epsilon$-net made up of a finite number of simplicial mappings. By counting this number carefully, we obtain

TheOREM 4. For each $\epsilon>0$, and $\delta>0$ with $\omega(\delta)=\epsilon$, and $m=d(\delta, X)$,

$$
H\left(\epsilon, D_{m n}\right) \leqq H(\epsilon, y)(m+1) 2^{H(\delta, X)} .
$$

Finally, $D_{m n}$ also contains an $\epsilon$-net made up of polynomial mappings of bounded degree:

THEOREM 5. For every $g \in D_{m n}$ there exists a vector-valued polynomial $p: R^{2 m+1} \rightarrow R^{2 n+1}$ of total degree $k$, such that, on $X_{m}$

$$
d(g, p) \leqq \text { const } \omega(2 m+1 / k) .
$$

The dimension of the space of these vector-valued polynomials provides us with an upper bound for the $\epsilon$-dimension of $D$ :

THEOREM 6. For each $\epsilon>0$ we have

$$
d\left(\epsilon, D_{m n}\right) \leqq \operatorname{const}(2 n+1)(1+1 / \epsilon)^{2 m+1} .
$$

These results give us estimates for the size of $D$ in terms of data 
taken from $X$ and $Y$. In many cases of practical interest estimates for these data are already available to us. Thus, for instance, if $X$ and $Y$ consist of signals which can be described as functions $x(t)$ of time $t$ satisfying certain conditions of boundedness and smoothness, then estimates for the entropy, capacity, and dimension of $X$ and $Y$ can be obtained from the work of Kolmogorov and Tihomirov [6]. In particular, if, for some $p, q \geqq 1$, the signals $x(t)$ in $X$ satisfy

$$
\begin{aligned}
& \int_{-\infty}^{+\infty}|f(t)|^{2}\left(1+t^{2}\right)^{p} d t \leqq 1, \\
& \int_{-\infty}^{+\infty}|\hat{f}(k)|^{2}\left(1+k^{2}\right)^{q} d k \leqq 1,
\end{aligned}
$$

where $\hat{f}(k)$ denotes the Fourier transform of $f(t)$, then we may introduce the effective time width $T(\epsilon)=(1 / \epsilon)^{1 / p}$ and the effective band width $W(\epsilon)=(1 / \epsilon)^{1 / q}$, and obtain

$$
\text { const } \begin{aligned}
T(\epsilon) W(\epsilon) & \leqq d(4 \epsilon, X) \\
& \leqq C(2 \epsilon, X) \leqq H(\epsilon, X) \\
& \leqq d(\epsilon, X) \log 4(1+1 / \epsilon) \\
& \leqq \text { const } T(\epsilon) W(\epsilon) \log (1+1 / \epsilon)
\end{aligned}
$$

thereby describing the complexity of such systems in terms of the physical constraints imposed on the available signals [8].

\section{REFERENCES}

1. R. T. Prosser, On the analysis and synthesis of certain abstract systems, Trans. Amer. Math. Soc. (submitted); Determinable classes of channels. II, J. Math. Mech. (submitted).

2. R. T. Prosser and W. L. Root, Determinable classes of channels. I, J. Math. Mech. 16 (1966), 365-397. MR 34 \#4052.

3. W. Hurewicz and H. Wallman, Dimension theory, Princeton Math. Series, vol. 4, Princeton Univ. Press, Princeton, N. J., 1941. MR 3, 312.

4. See reference 3.

5. A. N. Kolmogorov, Über die beste Annaherung von Funktionen einer gegebenen Funktionenklasse, Ann. of Math. (2) 37 (1936), 107-111.

6. A. N. Kolmogorov and V. M. Tihomirov, e-entropy and e-capacity of sets in function spaces, Uspehi Mat. Nauk 14 (1959), no. 2 (86), 3-86; English transl., Amer. Math. Soc. Transl. (2) 17 (1961), 277-364. MR 22 \#2890; MR 23 \#A2031.

7. R. T. Prosser, Note on metric dimension, Proc. Amer. Math. Soc. 25 (1970), 763765.

8. Presented to the National Academy of Sciences at Dartmouth College on October 13, 1969.

Dartmouth College, Hanover, New Hampshire 03755 